\title{
The changing medical research scene
}

\section{Louis-Philippe Boulet MD FRCPC, President, Canadian Thoracic Society}

A lthough the basic principles of medical research have not changed much over the past century, the ways that research is conducted has changed significantly, particularly over the past two decades. One aspect that has changed a great deal is the composition of research teams. In the past, researchers were working in isolation or in small groups, while today, multiple partnerships and large groups have become the rule. Furthermore, methods have progressed markedly, high technology is omnipresent and communications have achieved an unprecedented speed. The number of research works published has increased exponentially. This has created a strain for researchers having to balance the requirement for specialization with the need to cross discipline boundaries in research.

Mondialization has rapidly reached the medical scene, and research in particular. Collaboration between groups of researchers from different universities, within Canada and from other countries, has allowed fruitful exchanges of expertise, and national and/or international institutions or organizations have facilitated the networking of research groups. Also, efforts are being made to have scientists from different domains work together, and although there may be difficulties in regard to their respective "specialized culture or technical language", these collaborations may lead to new ways of approaching difficult problems and innovative ways of investigating or treating them. Not only have collaborations between different academic milieux been fruitful but, increasingly, joint university-industry initiatives have flourished. New modes of linking researchers and caregivers have been developed, particularly to help identify specific research targets and to develop ways to translate findings into patient care, adding further dimensions to the research process.
It is in this context that the recently formed Institute of Circulatory and Respiratory Health (ICRH), part of the Canadian Institutes of Health Research, has initiated its activities. The Institute aims to support research into the causes, prevention, screening, diagnosis, treatment support systems and palliation for a wide range of conditions affecting the heart, lung, brain, blood and blood vessels. This research will be done in partnership with other organizations, such as the Canadian Lung Association and the Canadian Thoracic Society. However, the nature of this partnership may take different forms, including joint support to the following: investigator-generated research (operating grants) and projects related to targeted 'priority' areas of research; regionally based training centres providing an infrastructure and mentorship to support the training of fellows and/or graduate students; fellowships in the respiratory field; multicentre networks working on a common theme (group grants); and interinstitute initiatives. Announcements of various programs have already been made, with more to come, and discussions with provincial lung associations should lead to a definition of the terms of agreement for these joint ICRH-Canadian Lung Association/Canadian Thoracic Society programs. You can check those developments on the Canadian Institutes of Health Research Web site (www.cihr.ca). 


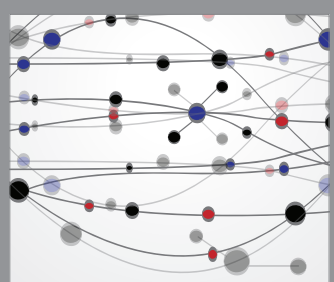

The Scientific World Journal
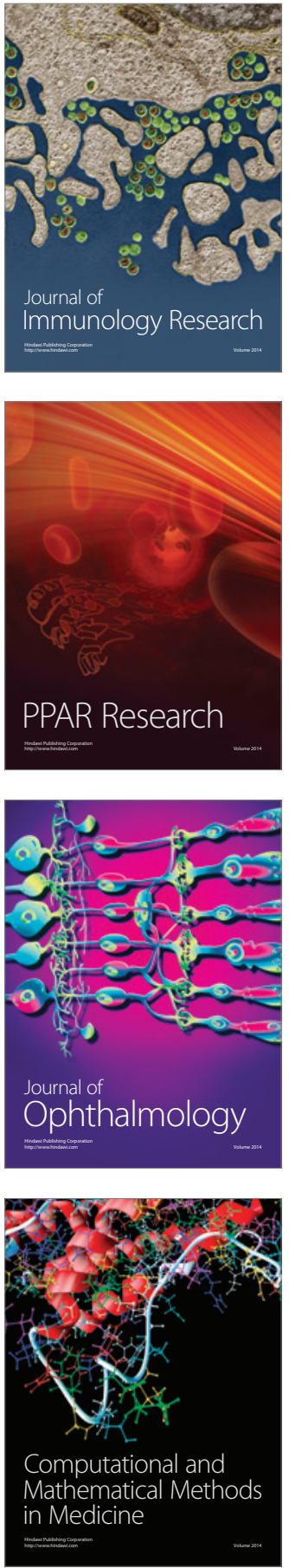

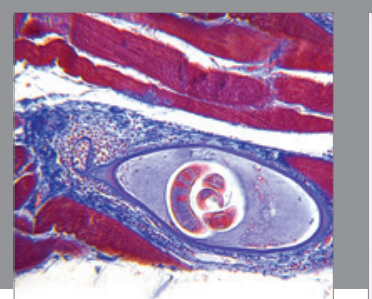

Gastroenterology Research and Practice

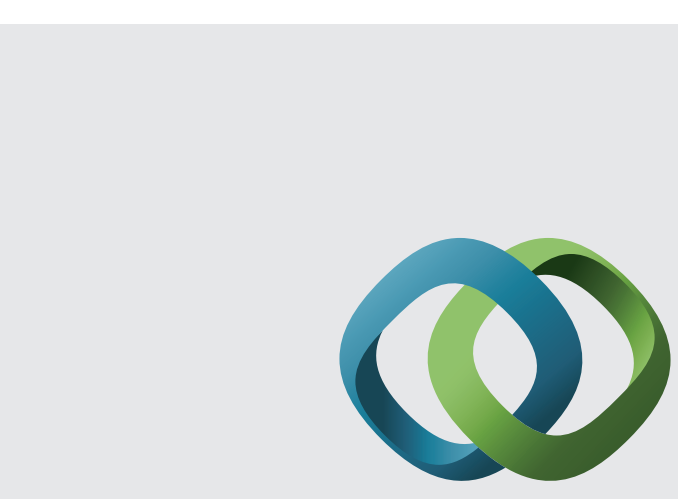

\section{Hindawi}

Submit your manuscripts at

http://www.hindawi.com
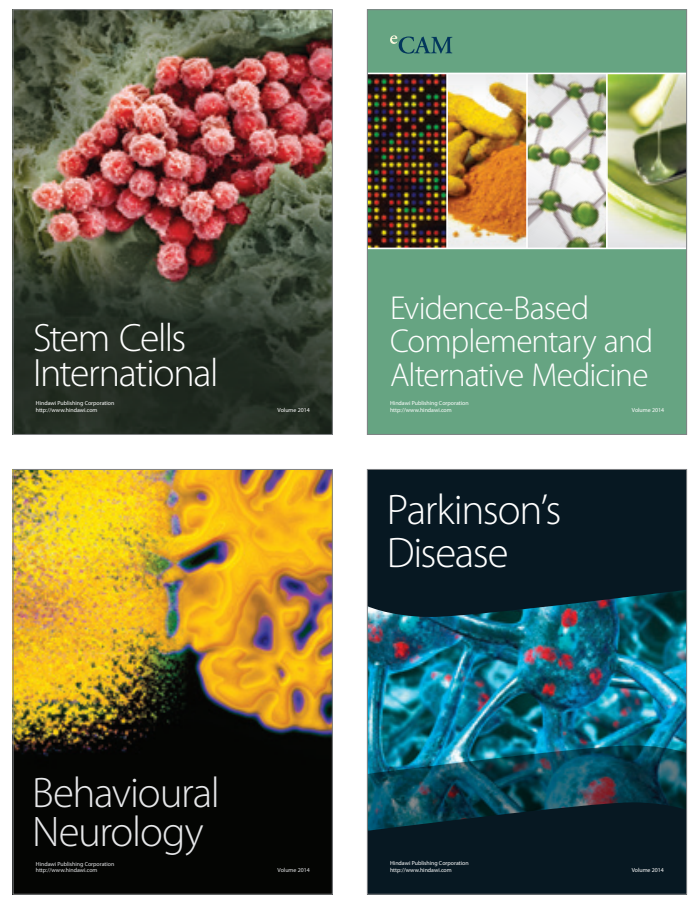
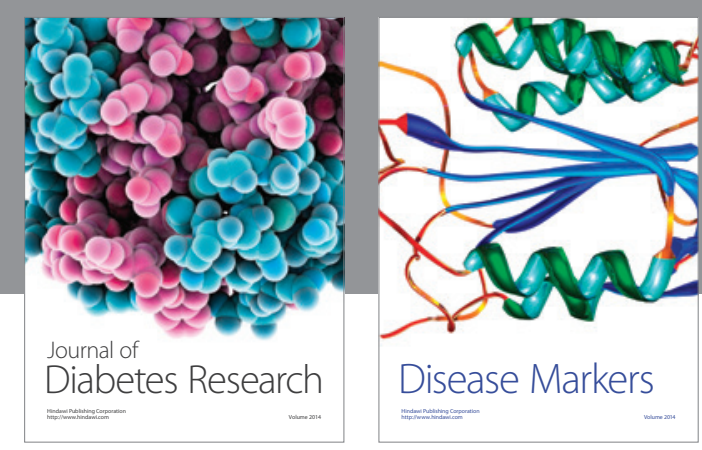

Disease Markers
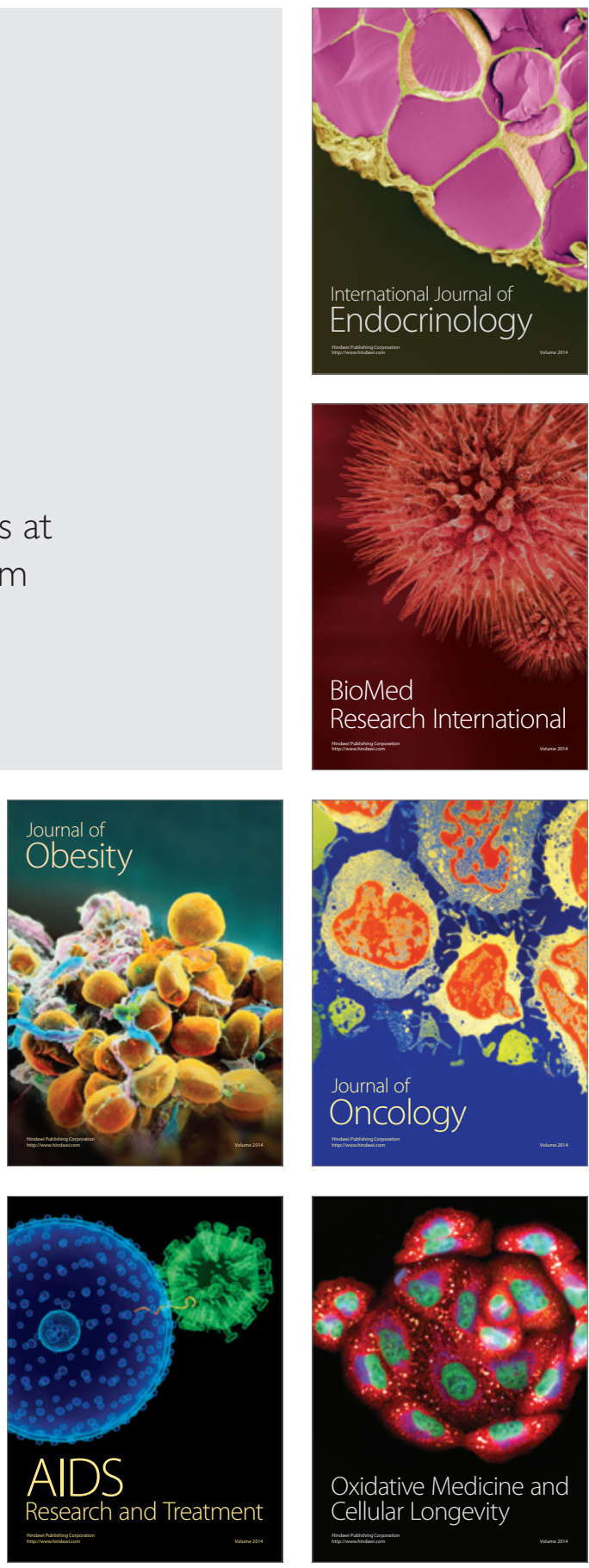